\title{
Methodology of designing integrated technological processes for manufacturing CNC machined parts
}

\author{
Viktor I. Guzeev ${ }^{1}$, and Danil Yu. Pimenov ${ }^{1, *}$ \\ ${ }^{1}$ Department of Automated Mechanical Engineering, South Ural State University, Lenin Prosp. 76, \\ Chelyabinsk 454080, Russia
}

\begin{abstract}
The article presents a new approach to the design of technological processes of processing parts on metal cutting machines in the integrated production conditions based on the expected forecast of the parts processing accuracy. The stages of choosing the parameters of processing steps are combined with determining the parameters of the cutting tool and machining attachments by simulation modeling. The design sequence begins with the first operation.
\end{abstract}

\section{Main part}

Mechanical engineering faces the task of ensuring an increase in the accuracy of parts manufacturing [1-4]. It is about a 10-times accuracy increase and ensuring the roughness of up to $\mathrm{Rz}=0.001 \mathrm{~mm}$. Alongside with that, the approaches to the design of technological processes of parts manufacturing on metal cutting machines remain mainly conventional. In the practice of technological design, in order to assess the processing accuracy by steps, technologists mainly use reference data. They specify the potential accuracy and the number of steps for a given method and equipment, however, they do not specify at what parameters of the tool, with what machining attachments and cutting modes it is possible to achieve them. This is largely due to the fact that the reference data are based on statistical data obtained at different enterprises and under different processing conditions, the parameters of which are generally not specified. The reference books for choosing the cutting modes do not fully trace the relationship with all the processing accuracy parameters. The choice of the cutting modes is often connected with the durability and strength of the cutting tool [5-9]. The absence of clear normative data on the integrated connection of all the processing accuracy parameters with the cutting modes, the parameters of the tools, machining attachments and equipment leads to a high role of subjective solutions in the design of technological processes. Therefore, the problems of ensuring accuracy are generally transferred to the stage of debugging the technological process, which significantly increases its labour capacity and cost. It was to some extent justified for mass and large-scale production with differentiated technological processes.

\footnotetext{
* Corresponding author: danil $\mathrm{u} @$,rambler.ru
} 
Given the low-volume output of modern production with highly concentrated technological operations, this problem is rather acute.

Conventional methods of designing technological processes of machine parts manufacturing are built according to the linear principle of performing the main stages, wherein the parameters obtained at the previous stages are often used at each subsequent stage. Surface treatment plans that determine the route for processing workpieces are also built on linear (sequential) design principles. Design begins with the determination of the last processing step and then sequentially to the first one, observing the principle of a gradual refinement of the accuracy parameters by steps, mainly the size and roughness of the surface. The tasks of ensuring the accuracy parameters of the shape and location of the part surfaces are mainly solved by setting additional processing steps. Using this approach, the possibilities of reducing the number of steps and the labour intensity of processing are rather limited, and the attempts to solve this problem through the use of more precise equipment do not give the expected effect. Another drawback of the existing methods of designing technological processes is a consistent implementation of the stages of choosing methods and processing plans, cutting tools, cutting modes, machining attachments. At the same time, these stages are interrelated because, for example, the cutting modes depend on the tool parameters, and, in its turn, the durability and reliability (strength) of the cutting tool depends on the cutting modes. For example, when machining precise holes, the ability of the processing method to ensure the specified accuracy parameters largely depends on the correct choice of the machining attachments. However, the machining attachments are generally chosen after setting operations and steps. A large experience in the creation of methodologies for optimizing technological processes has been accumulated [10-13]. However, the optimization is generally subdivided into structural and parametric. There is a known mutual influence of the parameters and structure of the technological operation. The result does not ensure an optimal multi-step operation in general. This situation dictates the need to create new approaches to the principles of designing a multi-step processing technology.

The Department of Automated Mechanical Engineering of the South Ural State University is engaged in developing such approaches. The basis of such approaches is formed by the principle of integrating the stages of designing technological processes. It consists in that the design begins with the first processing step, setting of the next step depends on achieving the highest possible accuracy in the given conditions at the previous step and in combining the stages of choosing the cutting tool, machining attachments and cutting modes with the choice of the processing step. The processing step can be performed both within the limits of one processing method, and with a change of the method at each step. In the first case, the problem is reduced to determining the required number of processing steps of the chosen method [14]. In the second case - to determining the plans for parts surface processing using different methods [15]. In this case, the interdependence of the design stages and their complex influence on the processing accuracy is taken into account. The stage of computer debugging of the technological process is included as a process design stage.

Such approach poses heightened requirements to mathematical models of the accuracy forecast, the content and form of presenting reference materials. Mathematical modelling should provide a comprehensive forecast of processing errors. The accumulated long-term experience in the creation of mathematical models of forecasting processing errors does not allow yet to combine them into an integrated complex analytical model that takes into account the mutual influence of many parameters of operations and steps on the processing accuracy. Today, in our opinion, it is expedient to use simulation modelling of technological operations, which is based on the joint use of a group of private mathematical 
models. Such approach allows you to synchronize (combine) stages of choosing the processing method, the purpose of the cutting tool and the machining attachments.

The basis of the procedure of design debugging of the technological process is formed by mathematical models of shaping work surfaces. The main task of debugging is to evaluate the options of processing steps in conjunction with the machining attachments and tools used. This problem is solved by simulation modelling.

Reference materials should correspond to the designed multi-step technological operations by the number of considered parameters.

General machine-building standards for cutting conditions developed at the department [16] have a technological focus. For the first time, they contain the data to determine the required number of processing stages, the minimum required depths of cutting and feeding for each processing stage, depending on the accuracy of workpieces (or semi-finished products), the accuracy reached after each tool travel (processing stage), as well as on several other technological limitations: rigidity of the workpiece and the tool, precision and rigidity of machines, etc. The reference book of cutting modes [17] contains a detailed explanation of choosing the required number of processing stages depending on the power loading of the technological system. Currently, work is underway to refine these data and change the form of their presentation to ensure computer design.

We will apply the mathematical logic apparatus to develop mathematical models of choosing the attachments [15]. As exemplified by choosing an auxiliary tool for holemaking operations, it is necessary to combine the requirements to individual equipment parameters resulting from the requirements of ensuring the accuracy at the designed processing step with the structural solutions of the machining attachments. The array of the requirements consists of the following:

- Disable linear movements in the tool retention unit;

- Enable linear movements in the tool retention unit;

- Disable annular movements of the auxiliary tool;

- Enable annular movements of the auxiliary tool;

- Increase the structural rigidity of the tool;

- Apply the front direction of the tool;

- Apply the rear direction of the tool;

- Apply the lash-free direction of the tool;

- Disable linear movements of the workpiece retention unit;

- Enable linear movements of the workpiece retention unit;

- Disable annular movements of the workpiece;

- Enable annular movements of the workpiece;

- Disable linear movements of the tool direction unit;

- Enable linear movements of the tool direction unit;

- Disable annular movements of the tool direction unit;

- Enable annular movements of the tool direction unit.

Each requirement for a specific type of attachments, in its turn, is provided by one or more technical solutions. Thus, to ensure each accuracy parameter, it is possible to create a system of requirements to machining attachments, which ultimately leads to a specific set of technical solutions and to specific types of attachments by choosing them from the technical solutions fund. For this purpose, a system of predicate equations is built.

The new methodology of the parametric design of hole-making plans is based on the following methods of ensuring accuracy:

- Comprehensive expected forecast of hole-making errors based on the mathematical models of hole formation; 
- Design process for debugging the step parameters to achieve the highest possible accuracy based on the established patterns of the influence of various processing parameters on the accuracy parameters;

- Formalized choice of machining attachments according to the accuracy requirements based on logical models.

The methodology of designing hole-making plans consists of the following stages.

- Stage 1 - The first processing step is chosen from the database of processing methods. Its choice is influenced by the type of a workpiece, the type of production, the route of the part processing, etc.

- Stage 2 - A set of machining attachments is formed to ensure the specified accuracy parameters at this step. For this purpose, a system of predicate equations is built. To implement this stage, a database of attachments is developed, indicating the necessary dimensional and other parameters.

- Stage 3 - Formation of the initial step parameters to calculate processing errors by the mathematical models of shape formation. The parameters of attachments, cutting modes, equipment, etc. are set based on the conditions of ensuring the maximum accuracy at each step. In this case, the output data of the steps taking into account the inheritance of errors form the input data for the subsequent processing steps. The data of the steps is formed taking into account the restrictions on durability, productivity, equipment capability, etc.

- Stage 4 - Calculation of processing errors by the mathematical models of shape formation. The maximum accuracy is reached on the basis of simulation modelling, including computer debugging of the step parameters. The stages of choosing the tool's attachments and cutting modes are combined.

- Stage 5 - The evaluation of ensuring the set accuracy parameters enables to decide on the further development of processing plans. If the set accuracy is ensured, the technological documentation is prepared with the indication of all the necessary step parameters. If one or more accuracy parameters are not fulfilled, the next step is set and the design process is repeated. Such cyclic design allowed us to control processing errors by the steps by varying the design and technological parameters to minimize the number of steps.

- Stage 6 - If the set accuracy parameters are not reached, the next step is set. In this case, the output data of the previous step is the input data of the next step.

- Stage 7 - If all the possibilities to reach the set processing accuracy are at the end in the given conditions, for example, the shape accuracy is set very "strictly", further steps are set using the finishing processing methods: jig boring, grinding, etc.

- Stage 8 - Final choice of the processing plan variant and the set of attachments. In this case, economic, organizational, technical and other indicators are taken into account.

This approach to design in comparison with the conventional approaches allows us to reduce the number of steps in up to 2 times, the production engineering costs are $\mathbf{2 0 - 3 0 \%}$ less, and the number of defective products is up to 3 times less.

\section{References}

1. S. Bratan, E. Vladetskaya, A. Kharchenko, Improvement of quality of details at round grinding in the conditions of a floating workshop, MATEC Web Conf. 129, 01083 (2017)

2. V.I. Guzeev, Feed speed control to assure accuracy of complex profile surfaces processing, Procedia Eng. 129, 495-499 (2015)

3. M. Mia, M.A. Khan, N.R. Dhar, Study of surface roughness and cutting forces using $A N N, R S M$, and ANOVA in turning of Ti-6Al-4V under cryogenic jets applied at flank 
and rake faces of coated WC tool, Int. J. Adv. Manuf. Technol. 93(1-4), 975-991 (2017)

4. A.M. Kozlov, A.A. Kozlov, Y.V. Vasilenko, Modeling a Cylindrical Surface Machined by a Non-circular Face Tool, Procedia Eng. 150, 1081-1088 (2016)

5. J.S. Dureja, V.K. Gupta, V.S. Sharma, M. Dogra, M.S. Bhatti, A review of empirical modeling techniques to optimize processing parameters for hard turning applications, Proc. Inst. Mech. Eng. Pt. B J. Eng. Manuf. 230(3), 389-404 (2016)

6. G. Królczyk, M. Gajek, S. Legutko, Effect of the cutting parameters impact on tool life in duplex stainless steel turning process | [Utjecaj parametara obrade na vijek alata $u$ procesu tokarenja dupleks čelika otpornog na koroziju], Teh. Vjesn. 20(4), 587-592 (2013)

7. S.N. Grigoriev, M.A. Volosova, V.L. Gurin, A.E. Seleznev, Wear of replaceable indexable inserts made of mixed cutting ceramics CC650 as a function of force parameters of steel ShKh15 face milling, J. Fric. Wear 36(6), 521-527 (2015)

8. G.M. Krolczyk, P. Nieslony, S. Legutko, Determination of tool life and research wear during duplex stainless steel turning, Arch. Civ. Mech. Eng. 15(2), 347-354 (2015)

9. N.E. Karkalos, A.P. Markopoulos, Applicability of ANN models and Taguchi method for the determination of tool life in turning, MATEC Web Conf. 112, 06005 (2017)

10. Á.R. Machado, A.E. Diniz, Tool wear analysis in the processing of hardened steels, Int. J. Adv. Manuf. Technol. 92(9-12), 4095-4109 (2017)

11. P.P. Pereverzev, D.Yu. Pimenov, Optimization of control programs for numerically controlled machine tools by dynamic programming, Russ. Eng. Res. 35(2), 135-142 (2015)

12. P.P. Pereverzev, A.V. Akintseva, Optimal internal grinding cycles in multidimensional control-parameter space, Russ. Eng. Res. 36(11), 974-978 (2016)

13. A. Akintseva, P. Pereverzev, Complex optimization of parameters for controlling the cycle of internal grinding by the method of dynamic programming, MATEC Web Conf. 129, 01019 (2017)

14. A.T. Abbas, D.Yu. Pimenov, I.N. Erdakov, T. Mikolajczyk, E.A. El Danaf, M.A. Taha, Minimization of turning time for high-strength steel with a given surface roughness using the Edgeworth-Pareto optimization method, Int. J. Adv. Manuf. Technol. 93(58), 2375-2392 (2017)

15. V.I. Guzeev, Theory and methodology of calculating the productivity of contour machining of parts of various accuracy on turning and milling CNC machines, Abstract of a thesis of the Doctor of Engineering Sciences. Chelyabinsk: Publishing House of the SUSU, 33 (1994).

16. I.P. Deryabin, Methodology of the parametric design of multi-pass processing of round holes using end measuring tools, Abstract of a thesis of the Doctor of Engineering Sciences. Chelyabinsk: Publishing House of the SUSU, 36 (2009).

17. General machine-building standards of cutting time and modes for the standardization of works performed on universal and multipurpose CNC machines. Part 2. Standard cutting modes, Moscow: Economics, 472 (1990)

18. V.I. Guzeev, V.A. Batuev, I.V. Surkov, Cutting modes for turning and drilling-milling and boring CNC machines: Reference book / Edited by V.I. Guzeev, M: Mashinostroenie (Mechanical Engineering), 368 (2005) 